\title{
Medikamentöse Behandlung der Depression im Alter - Empfehlungen aus Internationalen Leitlinien und aktueller Literatur
}

\section{Treating Late Life Depression with Antidepressants - A Summary of Recommendations in International Guidelines}

Autoren

Institute

\author{
K. E. Heilmann ${ }^{1}$, M. Wagner ${ }^{1,2}$, S. Riedel-Heller ${ }^{3}$, W. Maier ${ }^{1,2}$, F. Jessen ${ }^{4}$
}

Klinik und Poliklinik für Psychiatrie und Psychotherapie, Universitätsklinikum Bonn

2 Klinische Forschung, Deutsches Zentrum für Neurodegenerative Erkrankungen e.V., Bonn

Institut für Sozialmedizin, Arbeitsmedizin und Public Health (ISAP), Universität Leipzig, Medizinische Fakultät, Leipzig

${ }^{4}$ Klinik und Poliklinik für Psychiatrie und Psychotherapie, Universitätsklinikum Köln

Schlüsselwörter
Depression
Alter
Leitlinie
medikamentöse Behandlung
- Antidepressiva
Key words
antidepressants
antidepressant medication
depression
late life
elderly
guidelines

\section{Zusammenfassung \\ $\nabla$}

Depressive Erkrankungen im Alter zählen zu den häufigsten Erkrankungen und sind oft mit geringerer Lebensqualität und einem erhöhten körperlichen Erkrankungsrisiko verbunden. Die vorliegende Arbeit fasst pharmakologische Empfehlungen aktueller Leitlinien und Übersichtsarbeiten für diese spezielle Patientengruppe zusammen.

Die Behandlung mit Antidepressiva bei Depression im Alter $(65+)$ wird in den meisten Leitlinien empfohlen. Zur Wahl des Antidepressivums unter dem Aspekt möglicher Nebenwirkungen bzw. moderierender Faktoren liegen kaum Aussagen bzw. Empfehlungen vor.

Die spezifische Zielgruppe wird in den aktuellen Leitlinien unterschiedlich ausführlich betrachtet. $\mathrm{Zu}$ vielen Besonderheiten ihrer Behandlung liegen keine evidenzbasierten Behandlungsempfehlungen vor. Es besteht dringender Bedarf an aussagekräftigen Studien zu diesem Thema als Grundlage für zukünftige Leitlinienempfehlungen.

\section{Einführung \\ $\nabla$}

Der Anteil von älteren Menschen über 65 Jahre ist in den letzten Jahren gestiegen und wird in Zukunft weiter ansteigen. Waren 2008 noch $20 \%$ der deutschen Bevölkerung 65 Jahre oder älter, werden es 2060 34\% sein [1]. Auch in anderen Regionen der Welt wird ein deutlicher Anstieg in dieser Altersgruppe erwartet. Dabei ist die Zahl der körperlich und seelisch Erkrankten in dieser Bevölkerungsgruppe besonders hoch, etwa jeder Vierte ist körperlich erkrankt oder beeinträchtigt [2]. Bei den psychischen Erkrankungen, von denen etwa ein Viertel der über 65-Jährigen betroffen ist, gehören demenzielle und depressive Erkrankungen zu den häufigsten [3]. Eine neuere Metaanalyse ergab eine Punkt-Prävalenz von 7,2\% (93\% Cl 4,4\%-10,6\%) für eine Major Depression und eine Prävalenz ausge-

\section{Abstract \\ $\nabla$}

Late life depression is associated with severe health consequences, e.g. elevated risk of medical comorbidity and decreased quality of life.

This paper summarizes the recommendations of the international guidelines on evidence-based pharmacological treatment of depression in late life in a systematic review.

Pharmacological treatments for depression recommended by most of the guidelines, however, hardly address the issue of the possible side effects of antidepressants and other factors of multi-medication on the elderly.

Different guidelines pay different degrees of attention to the specific group of geriatric patients. There is a lack of evidence-based treatment recommendation that takes into consideration the specific age-related issues of sensitivity to adverse effects or pharmacokinetic interaction. Further research is required to provide a database for more refined recommendations in guidelines.

prägter depressiver Symptome von 17,1\% (95\% Cl $9,7 \%-26,1 \%$ ) bei über 75-Jährigen [4]. Aufgrund der hohen Prävalenz und der demografischen Entwicklung sind Depressionen im Alter von hoher Versorgungsrelevanz [5].

Depressionen im Alter führen zu einem erhöhten Risiko weiterer Erkrankungen und zu höheren Kosten für das Gesundheitswesen [6, 7]. Neben den kognitiven Störungen durch Altersdepressionen können auch soziale Fähigkeiten eingeschränkt sein. Menschen mit Depression im Alter haben ein erhöhtes Risiko der Selbstvernachlässigung und reduzierter Lebensqualität. Des Weiteren zeigen sich eine verkürzte Lebenserwartung und ein erhöhtes Suizidrisiko, insbesondere bei älteren Männern [5, 7 - 10].

Oft wird die Depression im Alter nicht erkannt, und wird sie erkannt, wird sie nicht ausreichend 
behandelt $[3,7,11]$. Doch Depressionen im Alter sind behandelbar und adäquate Versorgung führt neben einer Verbesserung der Lebensqualität zur Minderung der oben genannten Risiken [7, 8]. Die Wirksamkeit von antidepressiven Medikamenten bei der Behandlung von erkrankten Älteren wurde in einigen Metaanalysen und Reviews belegt [10, $12-14]$. Allerdings wird die Behandlung durch physiologische und kognitive Veränderungen, welche im Alterungsprozess auftreten, beeinflusst. Insbesondere sind Komorbiditäten und der veränderte Metabolismus bei der Behandlung älterer Depressiver zu beachten [9]. Zu den häufigsten Komorbiditäten zählen Herz-Kreislauf-Erkrankungen, Krankheiten des Bewegungsapparats, obstruktive Lungenerkrankungen und Diabetes [1]. Wechselwirkungen zwischen antidepressiver Medikation und Medikamenten zur Behandlung anderer Erkrankungen können zu Komplikationen führen, die es möglichst zu vermeiden gilt $[10,14]$.

Die medikamentöse Behandlung von depressiven Erkrankungen im höheren Lebensalter ist durch oben genannte Besonderheiten geprägt und erfordert deshalb spezifische Kenntnisse. Die vorliegende Arbeit stellt eine systematische Recherche aktueller Leitlinien zur Behandlung von Depression im Alter dar und gibt einen Überblick über die darin enthaltenen Empfehlungen zu medikamentösen Behandlungen der Depressionen im höheren Lebensalter.

\section{Methoden}

$\nabla$

\section{Literatursuche der Leitlinien}

Die Recherche von publizierten Leitlinien zu Depressionen erfolgte im März und April 2014 entsprechend den Empfehlungen des Deutschen Cochrane Zentrums (DCZ) in Kooperation mit dem Institut für medizinisches Wissensmanagement der AWMF (AWMF - IMWi) sowie dem Ärztlichen Zentrum für Qualität in der Medizin (ÄZQ) in spezifischen Leitliniendatenbanken und auf den Internetseiten ausgewählter Leitlinienentwickler (s. \& Tab. 1 [15]). Des Weiteren erfolgte eine Suche in den bibliografischen Datenbanken Medline und Embase, für die folgender Suchstring verwendet wurde: „(depression OR dysthymia OR (mood disorder) OR (affective disorder)) AND (elderly OR older OR (old age) OR (late life) OR geriatric)“. Die Ergebnisse wurden mithilfe von Suchfiltern (Practice Guideline, Consensus Development Conference) hinsichtlich methodischer Aspekte eingeschränkt.

\section{Auswahlkriterien der Leitlinien}

Es wurden folgende Einschlusskriterien festgelegt:

- Evidenz- und konsensbasierte Leitlinie

- Repräsentative Entwicklergruppe

- Vorliegen aggregierter Evidenz bzw. separater Behandlungsempfehlungen für die Behandlung älterer depressiver Menschen

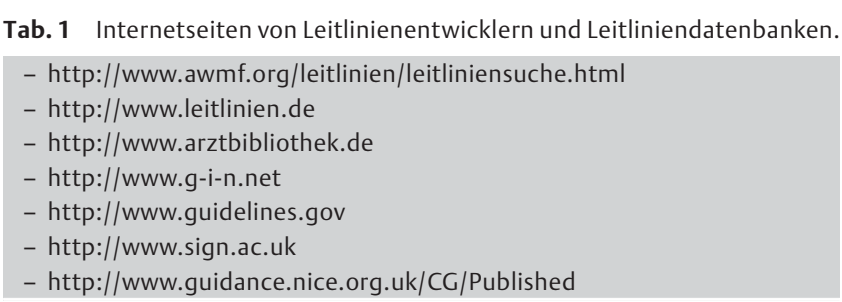

- Gültigkeit der Leitlinie, Erscheinungsdatum oder Update der

Leitlinie nicht älter als 5 Jahre (01/2009-04/2014)

- Redaktionelle Unabhängigkeit

- Deutsch- oder englischsprachig

\section{Literatursuche und Auswahlkriterien von Reviews und Meta-Analysen}

Da Leitlinien in ihrer Entstehung sehr zeitaufwendig sind, werden zwischen ihrer Entstehung und Veröffentlichung oft neuere Übersichtsarbeiten veröffentlicht. Diese können eine neue Ausrichtung des Themengebiets und gegebenenfalls Hinweise auf aktualisierte Leitlinien geben. Zur Ergänzung der Leitlinien um aktuelle Forschungsaggregate erfolgte im Juni und Juli 2014 eine Literaturrecherche zu aktuellen Meta-Analysen und Reviews bezüglich des Themas „Medikamentöse Behandlungsmöglichkeiten für depressive Erkrankungen im Alter“. Für die Recherche in der bibliografischen Datenbank MEDLINE wurde der Suchstring „(depression OR dysthymia OR (affective disorder) OR (mood disorder)) AND (elderly OR geriatric OR (late life) OR (old age)) AND (antidepressant OR (medical treatment) OR (pharmacological treatment)) AND (meta-analysis OR review)“" verwendet.

Die Recherche wurde hinsichtlich

- Aktualität (01/2010 - 07/2014),

- Methodik (nachvollziehbare systematische Recherche),

- Sprache (englisch- oder deutschsprachig)

- und des thematischen Bezugs (Behandlung von Altersdepressionen mit Medikamenten) eingeschränkt.

\section{Ergebnisse}

\section{Ergebnis der Leitlinienrecherche}

Die Literaturrecherche auf den Webseiten der Leitlinienentwickler und -datenbanken ergab 17 Treffer. Bei der Suche über andere Webseiten, Datenbanken und in den Literaturlisten wurden drei weitere Leitlinien identifiziert. Letztlich konnten sieben Leitlinien identifiziert werden, die den Einschlusskriterien entsprachen. Bei fünf Leitlinien war der Erscheinungszeitraum nicht aktuell. Die Methodik (evidenz- und konsensbasiert) war bei drei Leitlinien nicht erkennbar. Fünf Leitlinien enthielten keine pharmazeutischen Behandlungsempfehlungen oder spezifischen Empfehlungen für ältere Personen. Eine Übersicht der ausgeschlossenen Leitlinien und der Ausschlussgründe findet sich am Ende der Literaturangaben. Es folgt eine Auflistung der eingeschlossenen Leitlinien mit einer kurzen Beschreibung und den beteiligten Autoren (wie in den Leitlinien angegeben).

\section{Department of Veteran Affairs und Department of Defence (VA/DoD) 2009 \\ Management of Major Depressive Disorder [16]}

Die Leitlinie wurde unter der Schirmherrschaft der Veteran Health Administration (VHA), des Departments of VA und des DoD der Vereinigten Staaten, nach den Richtlinien des Departments of VA, entwickelt. Die Leitlinie gibt Empfehlungen für Behandler und Patienten mit einer Major Depression. Die Leitlinie enthält keine Empfehlungen für leichte Formen der Depression und Dysthymie und sie richtet sich an Erwachsene ab 18 Jahren. Ältere Patienten werden in verschiedenen Abschnitten (ohne Altersdefinition) angesprochen. Die Datengrundlage der Evidenzrecherche ist nicht summiert angegeben, über die ausführlichen Evidenztabellen lassen sich jedoch die verwendeten Quellen identifizieren. 
Arbeitsgruppe: C. Cassidy, T.J. Craig, M. Gerrity, L. A. Labbate, J. McQuaid, A. W. Muller, D. W. Oslin, J. A. Ray, T. Semla, J. W. Williams, A. M. Zeiss, L. Carchedi, C. Engel, D. Knitel, P.J. Lowry, G. H. Manos, E. C. McLaughlin, M. Ramos, S. K. Trice, R.J. Wilson, O. Susskind

Research Team: V.H. Coats, E. G. Erinoff, K. Schoelles, D. Snyder Healthcare Quality Informatics: M. D’Erasmo, R. Fishman, J. Marko

National Collaborating Centre for Mental Health (NCCMH) 2009a und 2009b

Die nachfolgend näher beschriebenen Leitlinien wurden durch das NCCMH entwickelt und durch das National Institute for Health and Clinical Excellence (NICE) beauftragt.

Depression in adults with a chronic physical health problem [17] Die Leitlinie enthält Empfehlungen zur Diagnostik und Behandlung von Depressionen bei komorbiden chronischen körperlichen Erkrankungen (z. B. Herz-Kreislauf-Erkrankungen oder Diabetes). Die Empfehlungen gelten für Erwachsene mit Depressionen unterschiedlicher Schweregrade. Aufgrund des gehäuften Auftretens chronischer Erkrankungen im Alter sind die Empfehlungen dieser Leitlinie auch für die Behandlung von Altersdepressionen relevant. In die Evidenzanalyse zur medikamentösen Behandlung von Depressionen (Altersuntergrenze bei Altersdepression nicht definiert) wurden 61 Studien einbezogen.

Arbeitsgruppe: D. Goldberg, S. Pilling, N. Andrews, V. Bird, F. Creed, C. Dowrick, M. Dyer, G. Grout, M. Haddad, J. Hindle, D. Kessler, K. Leggett, A. Lewis, R. Li, J. Lindesay, M. Ogden, S. Omarjee, J. Packham, C. Pettinari, M. Rizzo, R. Saunders, S. Stockton, C. Tayleo, D. Taylor, V. Thomas, S. Wilcox

Depression - The NICE Guideline on the Treatment and Management of Depression in Adults [18]

Die Leitlinie umfasst Empfehlungen zur Klassifizierung von Depressionen im Erwachsenenalter, Behandlungsempfehlungen für unterschiedliche Schweregrade und Betrachtungen zu Faktoren, die die Behandlung beeinflussen, wie zum Beispiel Nebenwirkungen von Interventionen oder von relevanten Patientenmerkmalen (z. B. Geschlecht oder Alter). Die Leitlinie umfasst keine Aussagen zur Diagnostik und Primärprävention von Depressionen. Depressive Erkrankungen im Alter (65 Jahre und älter) werden in einem separaten Abschnitt betrachtet, und in die Evidenzanalyse wurden 15 Studien einbezogen.

Arbeitsgruppe: I. Anderson, S. Pilling, A. Barnes, L. Bayliss, V. Bird, R. Burbeck, C. Chew-Graham, J. Clarke, M. Dyer, E. Flanagan, C. Harris, S. Hopkins, M. Kenwright, W. Kuyken, A. Lewis, G. Lewis, R. Li, B. Masterson, N. Meader, A. Meudell, A. Mitchell, R. Moore, S. Omarjee, C. Paton, A. Perez, P. Retsa, Mi. Rizzio, J. Robertson, R. Saunders, C. Sealey, B. Shackleton, T. Shackleton, S. sixsp;Stockton, C. Taylor, J. Wood

\section{American Psychiatric Association (APA) 2010}

Practice Guideline for the Treatment of Patients with

Major Depressive Disorder [19]

Unter Leitung des Lenkungsausschusses „Practice Guidelines“ und nach den Richtlinien „APA - Guideline Development Process“ wurde die Leitlinie zur Behandlung von Major Depression im Erwachsenenalter der APA entwickelt. Diese enthält neben Hintergrundinformationen zur Evidenz und Anregungen zu zukünftigem Forschungsbedarf allgemeine und spezifische Behandlungsempfehlungen für depressive Erkrankungen unter Berücksichtigung von beeinflussenden klinischen Merkmalen wie Alter oder
Geschlecht. Leichtere Depressionsformen werden in der Leitlinie nicht explizit angesprochen. Die Leitlinie spricht ältere Patienten ab 60 Jahren in verschiedenen Abschnitten an. Die Daten der Evidenzrecherche sind nicht summiert angegeben.

Arbeitsgruppe: A.J. Gelenberg, M.P. Freeman, J.C. Markowitz, J.F. Rosenbaum, M.E. Thase, M. H. Trivedi, R. S. Van Rhoads Independent Review Panel: V. I. Reus, J. R. DePaulo Jr., J. A. Fawcett, C. D. Schneck, D. A. Silbersweig

\section{Ministry of Health Singapore (MOH) 2012}

Depression - Clinical Practice Guidelines [20]

Die Leitlinie des Gesundheitsministeriums wurde von Vertretern verschiedener Gesundheitsberufe entwickelt und richtet sich mit ihren Empfehlungen vor allem an Ärzte, insbesondere an Hausärzte, aber auch an andere Gesundheitsberufe. Der Inhalt der Leitlinie umfasst Empfehlungen zur Behandlung und zum Management von Depressionen in der Schwangerschaft, in der Kindheit, im Erwachsenenalter und im Alter. Die Leitlinie umfasst keine Empfehlungen zu bipolaren Erkrankungen, psychotischen Depressionen oder Depressionen mit erhöhtem suizidalen Risiko. Altersdepressionen werden in einem separaten Kapitel (ohne Altersdefinition) betrachtet, dabei fehlt eine Angabe zu den Daten der Evidenzrecherche.

Arbeitsgruppe: C. Hong Choon, C. Lay Lin, C. F. Soon Leng, C. Kok Seng, D. Fung, H. Chen Yu, C. Swee Aun, K. Chai Ling, D. Kwek Seow Khee, W. Chua Pei Ling, L. Lim, J. Ling, T. Chay Hoon, P. Poh, T. Lay Ling, S. Kang, T. Yong Hui, A. Su Hsin Chuan, T. Woo Kheng, T. Bhing Leet, C. Yeo

Deutsche Gesellschaft für Psychiatrie, Psychosomatik und Nervenheilkunde (DGPPN) 2012 - Nationale Versorgungsleitlinie Unipolare Depression [21]

In Kooperation mit anderen Fachgesellschaften, unter anderem der Bundesärztekammer (BÄK), der Kassenärztlichen Bundesvereinigung (KBV) und der Arbeitsgemeinschaft der Wissenschaftlichen Medizinischen Fachgesellschaften e.V. (AWMF), entwickelte die DGPPN eine Leitlinie zur Diagnostik und Behandlung von Depressionen im Erwachsenenalter (ab 18 Jahren). Diese umfasst sowohl leichtere als auch schwerere Depressionen und richtet sich vor allem an Behandler (u. a. Ärzte und Psychotherapeuten) und Fachkrankenhäuser, aber auch an Betroffene und an Entscheidungsträger im Gesundheitswesen. Depressive Erkrankungen im Alter werden in einem kurzen Abschnitt (ohne Altersdefinition) betrachtet. Die der Evidenzrecherche zugrunde liegenden Daten sind nicht summiert angegeben.

Steuerungsgruppe: M. Härter, C. Klesse, I. Bermejo, M. Berger Arbeitsgruppe: A. Bleckmann, T. Bschor, J. Gensichen, T. Harfst, M. Hautzinger, C. Kolada, C. Kühner, J. Matzat, C. Mundt, W. Niebling, R. Richter, H. Schauenburg, F. Schneider, H. Schulz

Konsensgruppe: G. Adler, L. Adler, K. Bell, F. Bergmann, H. Böker, J. Finke, M. Franz, D. Haimerl, N. Hartkamp, I. Hauth, U. Hegerl, T. Heidenreich, G. Hildenbrand, W. Keller, J. Küchenhoff, C. Leiendecker, H.J. Luderer, H. Menzel, W. Merkle, R. Merod, N. Mönter, I. Neiser, A. Scharfenstein, U. Schweiger, R. Simon, G. Stoppe, D. Sturm, K. Tritt, C.P. Vogel, B. Waldherr, G. Wiedemann, T. Wiehn, M. Wolfersdorf

\section{Institut für Clinical System Improvement (ICSI) 2013}

Health Care Guideline: Adult Depression in Primary Care [22]

Das ICSI ist eine unabhängige, gemeinnützige Organisation zur Verbesserung der Gesundheitsfürsorge und entwickelte mit Hilfe einer Arbeitsgruppe, bestehend aus Ärzten, Psychologen, Kranken- 
schwestern, Apothekern und anderen relevanten Gesundheitsexperten die Leitlinie zur Behandlung von depressiven Erwachsenen (ab 18 Jahren). Die Leitlinie umfasst Empfehlungen zur Diagnose, Behandlung und Rückfallprophylaxe von schweren und anhaltenden depressiven Störungen. Altersdepressionen werden in einem separaten Kapitel für Patienten über 65 Jahren betrachtet, jedoch ohne summierte Angabe der Datengrundlage.

Arbeitsgruppe: J. Mitchell, M. Trangle, D. Kessler, S. Vincent, D. Rossmiller, B. Haight, T. Gabert, K. Somers, N. Mack, H. Novak, B. Degnan, E. Mallen, L. Setterlund

Neben diesen kurzen Zusammenfassungen wurden einige Qualitätsmerkmale zur Bewertung von Leitlinien nach dem Guidelines International Network (G-I-N) und dem deutschen Instrument zur methodischen Leitlinien-Bewertung (DELBI) in $\odot$ Tab. 2 zusammengestellt, um einen übersichtlichen Vergleich der inkludierten Leitlinien zu ermöglichen [23, 24]. Dabei wurden unter anderem die Zusammensetzung der Arbeitsgruppe, die Methodik und die Ergebnisgewinnung gewertet.

\section{Behandlungsempfehlungen der Leitlinien}

Der Umfang, in dem die einzelnen Leitlinien Empfehlungen zur medikamentösen Behandlung bei Depression im Alter geben, ist unterschiedlich. Einige Leitlinien beschränken sich auf kürzere Hinweise [16, 21], während andere ausführlichere Recherchen und Auswertungen für diese Patientengruppe angeben $[19,20]$. Die Empfehlungen und, soweit vorhanden, die Empfehlungsstärken zur medikamentösen Behandlung von depressiven Erkrankungen im Alter der ausgewählten Leitlinien werden in • Tab. 3 dargestellt. Im Allgemeinen werden medikamentöse Behandlungen mit Antidepressiva im Alter als wirksam [18, 20] oder als ähnlich wirksam wie im jüngeren Erwachsenenalter beschrieben $[21,22]$.

Zwischen den einzelnen Klassen von Antidepressiva, wie selektive Serotoninwiederaufnahmehemmer (SSRI), selektive Noradrenalinwiederaufnahmehemmer (SNRI), trizyklische Antidepressiva (TCA) und Monoaminooxidasehemmer (MAOH), wurden keine Wirksamkeitsunterschiede beschrieben $[18,20,21]$.

Unabhängig von diesem Befund werden SSRIs aufgrund geringerer Nebenwirkungen als erste Wahl gegenüber anderen Antide- pressiva empfohlen [16, 17, 20, 21]. Die Leitlinien der DGPPN und des $\mathrm{MOH}$ weisen jedoch darauf hin, dass bei SSRIs besonders auf Hyponatriämie und frequenzkorrigierte QT-Zeit-Verlängerung als Nebenwirkungen zu achten sei $[20,21]$.

Laut den Leitlinien besteht bei älteren depressiven Patienten generell eine erhöhte Sensitivität gegenüber Nebenwirkungen [18, $20]$, die deshalb genau zu überwachen sind [16, 18, 21, 22]. Ebenso kommen die meisten Leitlinien zu dem Schluss, die Behandlung bei depressiv erkrankten Älteren mit einer geringeren Anfangsdosis zu beginnen („start low, go slow“) und diese dann langsam zu steigern $[16,18,20,21]$, wobei nur eine Leitlinie eine klare Empfehlung dazu ausspricht und diese Dosierungsempfehlung besonders für körperlich komorbid Erkrankte ausspricht [20]. Die Leitlinie der DGPPN benennt dieses Vorgehen besonders bei der Behandlung mit TCAs [21]. Laut den Leitlinien der NCCHM sollte jedoch darauf geachtet werden, dass die Dosis nicht subtherapeutisch verabreicht wird $[17,18]$.

Die Wechselwirkungen mit anderen Medikamenten seien bei der Wahl des Antidepressivums ebenfalls zu beachten [19, 22]. Einige Leitlinien stellen zu diesem Zweck ausführliche Medikamentenlisten zu Wechselwirkungen und Nebenwirkungen der Antidepressiva mit Ausschlussgründen zur Verfügung $[16,17,21]$.

Die empfohlene Dauer der Behandlung sei ähnlich lang wie bei jüngeren Erwachsenen [19, 20], wobei die Leitlinien der NCCHM empfehlen, bei Älteren mit wiederholten depressiven Episoden die medikamentöse Behandlung dauerhaft aufrechtzuerhalten, um Rückfälle zu vermeiden [17, 18, 22]. Dafür sollte in der Stabilisierungsphase (nach etwa zwölf Monaten) mit der gleichen Dosis behandelt werden wie in der Initialphase [20].

Unterschiedliche Behandlungsempfehlungen nach Schweregrad der Depression: Hier wird bei schwerer Depression eine Kombination aus antidepressiver Medikation und Psychotherapie empfohlen [17, 20]. Auf die Behandlungen von mittelgradigen oder leichteren Episoden wird für Altersdepression nicht speziell eingegangen.

Eine weitere Option in der Behandlung ist die Augmentation mit Lithium. Dies wird in der NCCHM-Leitlinie (2009b) als nicht genügend evidenzbasiert bezeichnet. Die Behandlung mit Lithium sei mit deutlichen Risiken für depressive Ältere verbunden und

Tab.2 Qualitätsmerkmale der eingeschlossenen Leitlinien.

\begin{tabular}{|c|c|c|c|c|c|c|c|}
\hline $\begin{array}{l}\text { Herausgeber } \\
\text { (Jahr) }\end{array}$ & $\begin{array}{l}\text { VA/DoD } \\
\text { (2009) }\end{array}$ & $\begin{array}{l}\text { NCCMH } \\
\text { (2009a) }\end{array}$ & $\begin{array}{l}\text { NCCMH } \\
\text { (2009b) }\end{array}$ & $\begin{array}{l}\text { APA } \\
\text { (2010) }\end{array}$ & $\begin{array}{l}\text { MOH } \\
\text { (2012) }\end{array}$ & $\begin{array}{l}\text { DGPPN } \\
(2012)\end{array}$ & $\begin{array}{l}\text { ICSI } \\
(2013)\end{array}$ \\
\hline Land & USA & GB & GB & USA & Singapur & Deutschland & USA \\
\hline Quelle & [16] & [17] & [18] & [19] & {$[20]$} & [21] & [22] \\
\hline Zusammensetzung & MP & MP & MP & $(\mathrm{MP})^{1}$ & MP & MP & MP \\
\hline Arbeitsgruppe & & Patienten & $\begin{array}{l}\text { Patien- } \\
\text { ten }\end{array}$ & & Patienten & Patienten & Patienten \\
\hline Systematische Recherche & Ja & Ja & Ja & Ja & Ja & Ja & $(J a)^{2}$ \\
\hline $\begin{array}{l}\text { Systematische Ergebnis- } \\
\text { bewertung }\end{array}$ & $\mathrm{Ja}$ & $\mathrm{Ja}^{3}$ & $\mathrm{Ja}^{3}$ & $(\mathrm{Ja})^{4}$ & Ja & $\mathrm{Ja}$ & $\mathrm{Ja}$ \\
\hline Externe Begutachtung & $\mathrm{Ja}$ & Ja & $\mathrm{Ja}$ & $\mathrm{Ja}$ & Nein & $\mathrm{Ja}$ & Nein \\
\hline $\begin{array}{l}\text { Erklärung von Interessen- } \\
\text { konflikt und Finanzierung }\end{array}$ & Nein & $\mathrm{Ja}$ & $\mathrm{Ja}$ & $\mathrm{Ja}$ & Ja & $\mathrm{Ja}$ & Ja \\
\hline
\end{tabular}

VA/DoD = Department of Veterans Affairs/Department of Defence; APA = American Psychiatric Association; NCCMH = National Collaborating Centre for Mental Health; MOH = Ministry of Health Singapore; DGPPN = Deutsche Gesellschaft für Psychiatrie, Psychosomatik und Nervenheilkunde; ICSI = Institute for Clinical Systems Improvement; GB = Großbritannien; USA = Vereinigte Staaten von Amerika; Deutschl. = Deutschland; MP = Multiprofessionell, Ja = in der Leitlinie vorhanden, (Ja) = eingeschränkt vorhanden, Nein = unklar bzw. nicht angegeben.

${ }^{1}$ Hauptsächlich Ärzte, Psychotherapeuten und deren Fachgesellschaften, kein Pflegepersonal oder Ähnliches.

2 Nur in einer Datenbank (Medline).

${ }^{3}$ Zusätzlich Meta-Analysen von randomisierten kontrollierten Studien.

${ }^{4}$ Evidenzbewertungsschema nicht angegeben. 


\begin{tabular}{|c|c|c|c|c|c|c|c|}
\hline $\begin{array}{l}\text { Herausgeber } \\
\text { (Jahr) }\end{array}$ & $\begin{array}{l}\text { VA/DoD } \\
\text { (2009) }\end{array}$ & $\begin{array}{l}\text { NCCMH } \\
\text { (2009a) }\end{array}$ & $\begin{array}{l}\text { NCCMH } \\
\text { (2009b) }\end{array}$ & $\begin{array}{l}\text { APA } \\
(2010)\end{array}$ & $\begin{array}{l}\text { MOH } \\
\text { (2012) }\end{array}$ & $\begin{array}{l}\text { DGPPN } \\
(2012)\end{array}$ & $\begin{array}{l}\text { ICSI } \\
\text { (2013) }\end{array}$ \\
\hline Land & USA & GB & GB & USA & Singapur & Deutschland & USA \\
\hline Quelle & [16] & [17] & [18] & [19] & [20] & [21] & [22] \\
\hline $\begin{array}{l}\text { Wirksamkeit } \\
\text { (allgemein) }\end{array}$ & $\varnothing$ & $\varnothing$ & KK & $\varnothing$ & $* * *$ & KK & KK \\
\hline $\begin{array}{l}\text { Medikamen- } \\
\text { tenklasse }{ }^{1}\end{array}$ & $\varnothing$ & $\varnothing$ & KK & $\varnothing$ & $* * *$ & KK & $\varnothing$ \\
\hline SSRIs bevorzugt & KK & KK & $\varnothing$ & $\varnothing$ & $* * *$ & KK & $\varnothing$ \\
\hline $\begin{array}{l}\text { "start low, } \\
\text { go slow" }\end{array}$ & KK & $\varnothing$ & KK & $\varnothing$ & $* *$ & $\mathrm{KK}^{2}$ & $\varnothing$ \\
\hline \multicolumn{8}{|l|}{ Nebenwirkungen } \\
\hline Sensitivität & $\varnothing$ & $\varnothing$ & $\varnothing$ & $* * *$ & $* *$ & $\varnothing$ & $\varnothing$ \\
\hline Monitoring & KK & $\varnothing$ & KK & $\varnothing$ & $\varnothing$ & KK & KK \\
\hline $\begin{array}{l}\text { Wechsel- } \\
\text { wirkungen }\end{array}$ & $\varnothing$ & $\varnothing$ & $\varnothing$ & $* * *$ & $\varnothing$ & $\varnothing$ & $* * *$ \\
\hline $\begin{array}{l}\text { Behandlungs- } \\
\text { dauer }\end{array}$ & $\varnothing$ & $\mathrm{KK}^{3}$ & $\mathrm{KK}^{3}$ & $\mathrm{KK}^{4}$ & $\mathrm{KK}^{4}$ & $\varnothing$ & $\mathrm{KK}^{3}$ \\
\hline Augmentation & $\varnothing$ & $\varnothing$ & KK & $\varnothing$ & $\varnothing$ & $\varnothing$ & $\varnothing$ \\
\hline
\end{tabular}

$\mathrm{VA} / \mathrm{DoD}=$ Department of Veterans Affairs/Department of Defence; $\mathrm{APA}=$ American Psychiatric Association; $\mathrm{NCCMH}=$ National Collaborating Centre for Mental Health; $\mathrm{MOH}=$ Ministry of Health Singapore; DGPPN = Deutsche Gesellschaft für Psychiatrie, Psychosomatik und Nervenheilkunde; ICSI = Institute for Clinical Systems Improvement; GB = Großbritannien; USA = Vereinigte Staaten von Amerika; KK = Evidenzrecherche erfolgt, aufgrund mangelnder Studien nur klinischer Konsens ableitbar; *: Empfehlungsstärke C; ${ }^{* *}$ : Empfehlungsstärke B; ${ }^{* * *}$ : Empfehlungsstärke $A ; \varnothing=$ keine spezifische Aussage bzw. Empfehlung.

1 Kein Unterschied in der Wirksamkeit zwischen Wirkstoffgruppen.

${ }^{2}$ Bezogen auf die Behandlung mit trizyklischen Antidepressiva (TCA).

${ }^{3}$ Rückfallprophylaxe durch Weiterbehandlung mit Medikament.

${ }^{4}$ Behandlungsdauer gleich wie bei jungen Erwachsenen.

wird daher nicht empfohlen [18]. Des Weiteren beschreibt die Leitlinie der VA/DoD die Behandlung mit Psychostimulanzien wie Methylphenidat als mögliche Augmentationsstrategie für die Patientengruppe der körperlich schwer erkrankten Altersdepressiven, wobei dafür die wissenschaftliche Grundlage sehr unzureichend sei [16].

\section{Ergebnisse der Recherche nach aktuellen Reviews und Meta-Analysen ab 2010}

Die Recherche mit dem genannten Suchstring ergab 1065 Treffer. Nach erster Durchsicht konnte die Liste auf 114 Treffer begrenzt werden. Die 114 Ergebnisse wurden mithilfe der Einschlusskriterien überprüft. Bei 30 Treffern entsprach die Methodik nicht den gesuchten Anforderungen (Reviews oder Meta-Analysen). Des Weiteren berücksichtigten 44 Arbeiten keine älteren Patienten und bei 20 Treffern fehlte der thematische Bezug (medikamentöse Depressionsbehandlung). In die weitere Betrachtung konnten somit 20 Arbeiten einbezogen werden. Sieben der inkludierten Arbeiten sind Meta-Analysen [25-31], bei weiteren 13 Arbeiten handelt es sich um systematische Reviews. Die eingeschlossenen Reviews beziehen sich entweder ausschließlich auf randomisiert-kontrollierte Studien [32 - 36] oder zusätzlich auch auf pseudo- oder nicht-randomisierte Studien und andere Reviews [8, 37 - 43]. Die eingeschlossenen Studien werden nach Autoren alphabetisch gereiht in $\bullet$ Tab. 4 aufgeführt und kurz zusammengefasst.

\section{Inhalte der Reviews und Meta-Analysen}

Die gefundenen Arbeiten erbrachten grundsätzlich mit den Leitlinienempfehlungen übereinstimmende Ergebnisse. So kommen die Übersichtsarbeiten zur Effektivität von Antidepressiva in der Altersdepressionsbehandlung zu dem Ergebnis, das antidepressive Medikamente zur Behandlung von Altersdepressionen wirksam sind $[8,25,26,28,32$ - 36, 38, 39, 43]. In der Meta-Analyse von Te- deschini et al. (2011) konnte die Effektivität von diversen Antidepressiva für Patienten zwischen 55 und 65 Jahren nachgewiesen werden, nicht aber für Patienten über 65 Jahre. Die Autoren fordern für ein Verständnis dieses Phänomens weitere Untersuchungen und Vergleiche von verschiedenen Altersgruppen [25].

Der Vergleich verschiedener Wirkstoffklassen fällt ohne evidenzbasierte Präferenz aus [26, 29, 34, 36, 39, 41, 42]. Dabei sind drei Arbeiten zu beachten, die sich mit besonderen Wirkstoffen beschäftigen [34, 36, 42]. Zunächst ein cholinerg wirkender Stoff [42], der für Erwachsene Wirkung zeigt, nicht jedoch für alte Patienten, und des Weiteren ein selektiver Serotoninantagonist und -wiederaufnahmehemmer (SARI; 34), der eine ähnliche Wirkung wie TCA und SSRI zeigt. Eine weitere Arbeit betrachtet die Möglichkeit, Agomelatin als Antidepressivum einzusetzen. Für ältere Patienten wurde jedoch nur eine Studie gefunden, in der keine Wirksamkeit nachgewiesen werden konnte [36].

Einige Arbeiten befassen sich mit den Nebenwirkungen von Antidepressiva bei Depressionen im Alter und mit körperlichen komorbiden Erkrankungen. Die Verträglichkeit von selektiven SerotoninNoradrenalin-Wiederaufnahmehemmern (SSNRI) wird in einigen Arbeiten festgestellt [8, 28, 32, 39]. Zudem wird eine breitere Streuung von Nebenwirkungsphänomenen bei Altersdepression im Vergleich mit Depressionen im Erwachsenenalter aufgezeigt [8, 38]. Zu komorbiden Erkrankungen zeigt das Review von Katona, Bindman und Katona (2014), dass Antidepressiva zur Behandlung von Depressionen bei Alzheimer-Patienten eher ungeeignet sind [35]. Die Arbeit von Mitchell und Harvey fasst die Behandlungsempfehlungen bei häufigen komorbiden körperlichen Erkrankungen und deren Evidenzgrundlage übersichtlich zusammen. Diese Empfehlungen und ihre Evidenzbasis sind in 0 Tab. 5 dargestellt [36]. Zur Behandlung von Depressionen bei Koronarerkrankungen wird in der Arbeit von Pizzi et al. die Gabe von SSRI untersucht und als unbedenklich betrachtet. Die Autoren verweisen jedoch auf eine schwache Studienlage [30]. Arzneimittel, die insbesondere 
Tab. 4 Übersicht aktueller Reviews und Meta-Analysen zur pharmakologischen Behandlung von Altersdepressionen.

\begin{tabular}{|c|c|c|c|}
\hline $\begin{array}{l}\text { Autor/en } \\
\text { (Quelle) } \\
\text { Jahr }\end{array}$ & $\begin{array}{l}\text { Review/ } \\
\text { Meta-Analyse }\end{array}$ & $\begin{array}{l}\text { Thema der Arbeit } \\
\text { (untere Altersgrenze in Jahren) }\end{array}$ & Ergebnisaussagen \\
\hline $\begin{array}{l}\text { Boyce et al. } \\
{[43]} \\
2012\end{array}$ & R & $\begin{array}{l}\text { Effektivität von Antidepressiva bei } \\
\text { Pflegeheimpatienten (65) }\end{array}$ & Antidepressiva sind wirksam gegenüber Placebo \\
\hline $\begin{array}{l}\text { Cooper et al. } \\
\text { [37] } \\
2011\end{array}$ & R & Behandlung von Therapieresistenz (55) & $\begin{array}{l}\text { Bei nicht erfolgreicher Behandlung mit einem } \\
\text { Antidepressivum führt ein Medikamentenwech- } \\
\text { sel oder ein zusätzlicher Wirkstoff bei } 50 \% \text { der } \\
\text { Betroffenen zum Behandlungserfolg; } \\
\text { Augmentation mit Lithium ist wirksam bei re- } \\
\text { fraktären Depressionen }\end{array}$ \\
\hline $\begin{array}{l}\text { Del Casale et al. } \\
\text { [32] } \\
2012\end{array}$ & $\mathrm{R}$ & Duloxetin (SSNRI) als Antidepressivum (k.A.) & $\begin{array}{l}\text { Duloxetin ist gut verträglich und wirksam } \\
\text { gegenüber Placebo }\end{array}$ \\
\hline $\begin{array}{l}\text { Dhillon } \\
\text { [33] } \\
2013\end{array}$ & $\mathrm{R}$ & Duloxetin (SSNRI) als Antidepressivum (k.A.) & $\begin{array}{l}\text { Duloxetin ist gut verträglich und wirksam ge- } \\
\text { genüber Placebo; keine Vergleiche mit anderen } \\
\text { Wirkstoffen }\end{array}$ \\
\hline $\begin{array}{l}\text { Diniz et al. } \\
{[8]} \\
2011\end{array}$ & R & $\begin{array}{l}\text { Neuere Entwicklungen zur Behandlung von } \\
\text { affektiven Störungen im Alter (k.A.) }\end{array}$ & $\begin{array}{l}\text { Antidepressiva, Phasenprophylaktika und ande- } \\
\text { re Medikamente sind trotz erwiesener Effektivi- } \\
\text { tät mit Risiken behaftet, für die neue Behand- } \\
\text { lungsstrategien entwickelt werden sollten }\end{array}$ \\
\hline $\begin{array}{l}\text { Dolder, Nelson, Stump } \\
\text { [38] } \\
2010\end{array}$ & R & $\begin{array}{l}\text { Pharmakologie, Effektivität und Sicherheit von } \\
\text { neueren Antidepressiva gegenüber Placebo (k.A.) }\end{array}$ & $\begin{array}{l}\text { Antidepressiva sind effektiv (geringer Effekt), } \\
\text { werden ähnlich toleriert wie von jüngeren } \\
\text { Erwachsenen, es gibt aber eine größere Streuung } \\
\text { von möglichen Nebenwirkungen; } \\
\text { zu einigen neueren Antidepressiva (Desvenlafa- } \\
\text { xin) gibt es noch keine spezifischen Ergebnisse } \\
\text { bei Altersdepression }\end{array}$ \\
\hline $\begin{array}{l}\text { Fagiolini et al. [34] } \\
2012\end{array}$ & $\mathrm{R}$ & $\begin{array}{l}\text { Einsatz von Trazodon (SARI) als Antidepressivum } \\
\text { (k.A.) }\end{array}$ & $\begin{array}{l}\text { Trazodon ähnlich wirksam wie TCA und SSRI, } \\
\text { weniger Nebenwirkungsneigung im Vergleich } \\
\text { zu SSRI }\end{array}$ \\
\hline $\begin{array}{l}\text { Holland, Bhogle } \\
\text { [39] } \\
2013\end{array}$ & $\mathrm{R}$ & $\begin{array}{l}\text { Vergleich von Sertralin (SSRI) und Mirtazapin } \\
\text { (SSNRI) (k.A.) }\end{array}$ & $\begin{array}{l}\text { Mirtazapin tendenziell zu bevorzugen, aber stark } \\
\text { abhängig von Komorbiditäten und bestehender } \\
\text { Medikation }\end{array}$ \\
\hline $\begin{array}{l}\text { Howland } \\
{[41]} \\
2011\end{array}$ & $\mathrm{R}$ & Agomelatin als Antidepressivum (k.A.) & $\begin{array}{l}\text { Kein Effekt bei der Behandlung von Alters- } \\
\text { depressionen im Vergleich zu Placebo }\end{array}$ \\
\hline $\begin{array}{l}\text { Jaffe, Novakovic, Peselow } \\
\text { [42] } \\
2013\end{array}$ & $\mathrm{R}$ & $\begin{array}{l}\text { Scopolamin als Antidepressivum im Vergleich } \\
\text { zu Placebo (62) }\end{array}$ & $\begin{array}{l}\text { Wirksamkeit für ältere Patienten konnte nicht } \\
\text { nachgewiesen werden (nur eine Studie), bei } \\
\text { jungen Patienten wirksam }\end{array}$ \\
\hline $\begin{array}{l}\text { Katona, Bindman, Katona } \\
\text { [35] } \\
2014\end{array}$ & R & $\begin{array}{l}\text { Effektivität, Sicherheit, komorbide Erkrankungen, } \\
\text { Therapieresistenz und Rezidivprophylaxe bei } \\
\text { Behandlung mit Antidepressiva (59) }\end{array}$ & $\begin{array}{l}\text { Antidepressiva sind effektiv, jedoch nur geringer } \\
\text { Unterschied gegenüber Placebo; Antidepressiva } \\
\text { nicht geeignet zur Behandlung bei depressiven } \\
\text { Alzheimer-Patienten; } \\
\text { Rezidivprophylaxe sinnvoll, wenn Behandlung } \\
\text { wirkungsvoll; } \\
\text { Forschungsbedarf für Behandlung bei Therapie- } \\
\text { resistenz }\end{array}$ \\
\hline $\begin{array}{l}\text { Kok, Nolen, Heeren } \\
{[26]} \\
2012\end{array}$ & $\mathrm{M}, \mathrm{R}$ & $\begin{array}{l}\text { Effektivität von Antidepressiva gegenüber } \\
\text { Placebo (55) }\end{array}$ & $\begin{array}{l}\text { Antidepressiva sind effektiv; } \\
\text { kein Unterschied zwischen Wirkstoffklassen, } \\
\text { dies gilt auch für schwere depressive Episoden }\end{array}$ \\
\hline $\begin{array}{l}\text { Kok, Heeren, Nolen } \\
{[27]} \\
2011\end{array}$ & $\mathrm{M}, \mathrm{R}$ & Rezidivprophylaxe (55) & $\begin{array}{l}\text { Eine Aufrechterhaltung ist effektiv gegenüber } \\
\text { Placebo zur Verhinderung von wiederkehrenden } \\
\text { Symptomen; } \\
\text { kein Unterschied zwischen TCA und SSRI }\end{array}$ \\
\hline $\begin{array}{l}\text { Maust, Oslin, Thase } \\
{[40]} \\
2013\end{array}$ & R & $\begin{array}{l}\text { Augmentation mit Lithium, Psychostimulanzien } \\
\text { und neueren Neuroleptika (62) }\end{array}$ & $\begin{array}{l}\text { Datengrundlage nicht eindeutig genug für evi- } \\
\text { denzbasierte Behandlungsempfehlung; } \\
\text { Augmentation mit verschiedenen Wirkstoffen } \\
\text { wirksam, bei teilweise erhöhtem Nebenwir- } \\
\text { kungsrisiko }\end{array}$ \\
\hline $\begin{array}{l}\text { Mitchell, Harvey } \\
\text { [36] } \\
2014\end{array}$ & $\mathrm{R}$ & $\begin{array}{l}\text { Epidemiologie und Behandlung unter Betrachtung } \\
\text { von Depressiven mit komorbiden körperlichen } \\
\text { Erkrankungen (k.A.) }\end{array}$ & $\begin{array}{l}\text { Bei den meisten komorbiden Erkrankungen soll- } \\
\text { te auf TCA verzichtet werden; } \\
\text { Datengrundlage für eine Empfehlung bei Morbus } \\
\text { Parkinson und COPD nicht ausreichend }\end{array}$ \\
\hline
\end{tabular}


Tab. 4 (Fortsetzung)

\begin{tabular}{|c|c|c|c|}
\hline $\begin{array}{l}\text { Autor/en } \\
\text { (Quelle) } \\
\text { Jahr }\end{array}$ & $\begin{array}{l}\text { Review/ } \\
\text { Meta-Analyse }\end{array}$ & $\begin{array}{l}\text { Thema der Arbeit } \\
\text { (untere Altersgrenze in Jahren) }\end{array}$ & Ergebnisaussagen \\
\hline $\begin{array}{l}\text { Oakes et al. } \\
{[28]} \\
2013\end{array}$ & M & Duloxetin (SSNRI) gegenüber Placebo (65) & $\begin{array}{l}\text { Duloxetin ist sicher und hat ähnlich wenig } \\
\text { Nebenwirkungen im Vergleich zu Placebo }\end{array}$ \\
\hline $\begin{array}{l}\text { Pizzi et al. } \\
{[30]} \\
2011\end{array}$ & M & $\begin{array}{l}\text { SSRI bei Patienten mit Depression und } \\
\text { Koronarerkrankungen ( } 56 \text { ) }\end{array}$ & $\begin{array}{l}\text { SSRI ungefährlich für depressive Koronarer- } \\
\text { krankte; } \\
\text { schwache Studiengrundlage }\end{array}$ \\
\hline $\begin{array}{l}\text { Seitz, Gill, Conn } \\
{[29]} \\
2010\end{array}$ & $M, R$ & $\begin{array}{l}\text { Vergleich von Citalopram mit anderen } \\
\text { Antidepressiva (65) }\end{array}$ & $\begin{array}{l}\text { Kein Unterschied zwischen Citalopram und } \\
\text { anderen Antidepressiva }\end{array}$ \\
\hline $\begin{array}{l}\text { Tedeschini et al. [25] } \\
2011\end{array}$ & M & $\begin{array}{l}\text { Effektivität von Antidepressiva im Vergleich } \\
\text { zu Placebo (55) }\end{array}$ & $\begin{array}{l}\text { Antidepressiva }(55+) \text { sind effektiv; } \\
\text { Hinweise darauf, dass die Effektivität mit } \\
\text { zunehmendem Alter }(65+) \text { geringer wird }\end{array}$ \\
\hline $\begin{array}{l}\text { Wilkinson, Izmeth } \\
\text { [31] } \\
2012\end{array}$ & $M, R$ & Chochrane Review zur Rezidivprophylaxe (60) & $\begin{array}{l}\text { Die Vorteile einer langfristigen antidepressiven } \\
\text { Behandlung sind nicht eindeutig, es kann keine } \\
\text { Empfehlung abgeleitet werden; } 12 \text { Monate Auf- } \\
\text { rechterhaltung der Medikation erscheint sinn- } \\
\text { voll, basierend auf einer sehr kleinen Datenlage. }\end{array}$ \\
\hline
\end{tabular}

COPD = chronisch obstruktive Lungenerkrankung; SSRI = selektive Serotoninwiederaufnahmehemmer; SSNRI = selektive Serotonin-Noradrenalin-Wiederaufnahmehemmer; TCA = trizyklische Antidepressiva; EKT = Elektrokrampftherapie; SARI = Serotoninantagonist und -wiederaufnahmehemmer; k.A. = keine Angabe

Tab. 5 Behandlungsempfehlungen für häufige komorbide Erkrankungen bei Altersdepressionen [vgl. 36].

\begin{tabular}{|c|c|c|}
\hline Erkrankung & $\begin{array}{l}\text { Zugrunde lie- } \\
\text { gende Evidenz }\end{array}$ & $\begin{array}{l}\text { Behandlungsemp- } \\
\text { fehlung }\end{array}$ \\
\hline Myokardinfarkt & Moderat bis stark & Vermeiden von TCA \\
\hline $\begin{array}{l}\text { Chronische } \\
\text { Herzinsuffizienz }\end{array}$ & Keine & Vermeiden von TCA \\
\hline $\begin{array}{l}\text { Zerebrovaskuläre } \\
\text { Erkrankungen }\end{array}$ & Limitiert & Vermeiden von TCA \\
\hline Krebserkrankungen & Limitiert & $\begin{array}{l}\text { Vermeiden von TCA bei } \\
\text { eingeschränkter Darm- } \\
\text { beweglichkeit; } \\
\text { Vermeiden von SSRI mit } \\
\text { Tramadol oder Tamoxifen }\end{array}$ \\
\hline $\begin{array}{l}\text { Chronische Nieren- } \\
\text { erkrankungen }\end{array}$ & Limitiert & $\begin{array}{l}\text { TCA können zu } \\
\text { Inkontinenz führen }\end{array}$ \\
\hline COPD & Limitiert & $\begin{array}{l}\text { Keine spezifische } \\
\text { Empfehlung möglich }\end{array}$ \\
\hline Morbus Parkinson & $\begin{array}{l}\text { Keine Evidenz für } \\
\text { Antidepressiva, } \\
\text { Pramipexole ist } \\
\text { effektiv }\end{array}$ & $\begin{array}{l}\text { Keine spezifische } \\
\text { Empfehlung möglich }\end{array}$ \\
\hline
\end{tabular}

COPD = chronisch obstruktive Lungenerkrankung; SSRI = selektive Serotoninwiederaufnahmehemmer; TCA = trizyklische Antidepressiva;

im Alter zu potenziellen Schädigungen führen können, werden als potenziell inadäquate Medikation (PIM) bezeichnet. Dazu zählen auch einige Antidepressiva, wie beispielsweise Amitriptylin [vgl. 44]. Eine Übersicht über Antidepressiva, die insbesondere im Alter zu unerwünschten Nebenwirkungen und Wechselwirkungen führen können, geben etablierte Screening-Tools. Dazu zählen die für den amerikanischen Markt etablierte Beers-Liste [45] sowie die auf den deutschen Arzneimittelmarkt zugeschnittene PRISCUS-Liste. [44]. Darüber hinaus wurden die sogenannten START/STOPPKriterien entwickelt, die Bezug nehmen auf Wechselwirkungen zwischen häufig verordneten Medikamente im Alter (Drug-DrugInteraction, DDI). Diese Wechselwirkungen stellen aus gerontopsychiatrischer Sicht aufgrund der Vielzahl an Komorbiditäten im
Alter ein häufiges Problem dar. STOPP steht für „Screening Tool of Older Person's Prescriptions“, START für „Screening Tool to Alert doctors to Right Treatment". So findet sich beispielsweise ein STOPP-Kriterium für ältere Patienten zur gleichzeitigen Einnahme von SSRI und NSAID [46, 47].

Mit der Behandlung zur Rezidivprophylaxe und Therapieresistenz beschäftigen sich vier der gefundenen Übersichtsarbeiten. Cooper et al. (2011) kamen zu dem Ergebnis, dass nach der nicht erfolgreichen Behandlung mit einem Antidepressivum ein zusätzlicher Wirkstoff oder ein Medikamentenwechsel in $50 \%$ der Fälle zu einem Behandlungserfolg führt. Besonders die Wirksamkeit einer Augmentation mit Lithium wird von den Autoren aufgezeigt. Insgesamt bemängeln sie eine unzureichende Studiengrundlage [37]. Im aktuellen Review von Katona, Bindman und Katona (2014) wird ebenfalls auf eine unzureichende Erforschung von Therapieresistenzen bei Altersdepressionen verwiesen und im Bereich der Rezidivprophylaxe eine fortgesetzte Medikation empfohlen, sofern die Medikation bisher wirksam war [35]. Zu diesem Schluss kommen auch Kok, Heeren und Nolen (2011), die in ihrer Arbeit zur Rezidivprophylaxe keinen Unterschied zwischen TCA und SSRI feststellen konnten, jedoch eine Überlegenheit von Antidepressiva gegenüber Placebo zur Verhinderung einer erneuten Symptomatik aufzeigen [27]. Das Chochrane-Review zur Rezidivprophylaxe kommt zu dem Ergebnis, dass die Vorteile einer langfristigen Behandlung mit Antidepressiva nicht eindeutig aus der bisherigen Forschung ableitbar sind, dass die vorhandene Datengrundlage jedoch eine Aufrechterhaltung über 12 Monate sinnvoll erscheinen lässt [31].

Zur Augmentation mit Lithium sei auf die bereits beschriebene Arbeit von Cooper und Kollegen verwiesen, die die Wirksamkeit einer Lithiumaugmentation feststellten [37]. Des Weiteren wurde ein Review von Maust, Oslin und Thase (2013) identifiziert, das sich neben einer Lithiumaugmentation mit Behandlungsergänzungen über Psychostimulanzien und neueren Neuroleptika befasst. Darin kommen die Autoren zu dem Ergebnis, dass die Datengrundlage zu diesen Behandlungsstrategien hauptsächlich aus Fallbeispielen und nicht randomisierten Studien besteht und dass keine evidenzbasierte Behandlungsstrategie abgeleitet wer- 
den kann. Besonders die Datenlage für Psychostimulanzien und Neuroleptika zeigt zwar Wirksamkeitstendenzen, jedoch mit höherem Nebenwirkungsrisiko [40].

\section{Diskussion}

Die untersuchten Leitlinien gehen von der Wirksamkeit antidepressiv wirkender Medikamente bei älteren Patienten aus, wobei kein Wirksamkeitsunterschied zwischen den Medikamentenklassen beschrieben wird. Zu diesem Ergebnis kommen auch die betrachteten Reviews und Meta-Analysen. Die verschiedenen Wirkstoffgruppen sind vor allem bei gleichzeitig bestehenden körperlichen Erkrankungen und Interaktionen mit deren Medikation zu beachten; dazu sei auf $\bullet$ Tab. 5 und die ausführlicheren Wirkstoff-Wechselwirkungstabellen einiger Leitlinien verwiesen $[16,17,21]$. Einige Wirkstoffe und Wirkstoffgruppen wie MAOH, SARI, SNRI, SSNRI, Scopolamin oder auch Agomelatin werden in den Leitlinien nicht näher betrachtet. Dies ist vermutlich auf die unzureichende Datengrundlage zurückzuführen und darauf, dass die Wirkstoffe keine besonderen Vorteile für Patienten mit Altersdepressionen bieten, so dass sie in die übergeordnete Perspektive der Leitlinien keinen Eingang gefunden haben könnten. Dies zeigt sich in den betrachteten Reviews und Meta-Analysen, die kaum Studien zu diesen Wirkstoffen bei älteren Patienten fanden und in den wenigen Ausnahmen keinen Wirkungsvorteil gegenüber SSRI und auch TCA auffinden konnten [34, 36, 42]. Weitere Studien auf diesem Gebiet könnten die Reaktionsmöglichkeiten auf spezifische Bedürfnisse wie Nebenwirkungssensibilität und körperliche Erkrankungen erweitern.

Die Leitlinien stimmen darin überein, dass bei einer medikamentösen Behandlung von depressiven Erkrankungen im Alter mit einer erhöhten Sensibilität für Nebenwirkungen zu rechnen und daher deren engmaschige Überwachung geboten ist, wobei keine eindeutigen Empfehlungen zu dem genauen Umfang der Überwachung gegeben werden. In der Regel sind SSRI aufgrund besserer Verträglichkeit und weniger Ausschlussgründen bei komorbiden Erkrankungen zu empfehlen, wobei jedoch insbesondere auf Hyponatriämie $[20,21]$ zu achten ist. Um die Sensibilität gegenüber den Nebenwirkungen in der Behandlung adäquat zu berücksichtigen, fehlen bislang jedoch aussagekräftige Studien, die die Nebenwirkungen der Medikamentenklassen systematisch miteinander vergleichen [13]. Des Weiteren wird in den meisten Leitlinien der Behandlungsansatz empfohlen, gering einzudosieren und langsam aufzudosieren („start low, go slow“). Dabei sollte aber eine therapeutisch wirksame Dosis nicht unterschritten werden $[17,18]$.

Die Leitlinien empfehlen eine zwölfmonatige Aufrechterhaltung der Medikation zur Rezidivprophylaxe und bei schweren depressiven Episoden eine Kombination mit Psychotherapie [17-20, 22]. Es ist zu beachten, dass die Empfehlungen zur Rezidivprophylaxe und zu verschiedenen Ausprägungen der Depression (leicht, mittelgradig, schwer) nicht in allen Leitlinien explizit aufgeführt sind. In diesem Fall wird nach der Empfehlung für depressive Erwachsene (bei leichten Episoden Psychotherapie und ab mittelgradiger Episode Kombination aus Psychotherapie und Medikation; zwölfmonatige Rezidivprophylaxe) behandelt. Es bleibt aber unklar, ob dies für Altersdepressionen evident ist oder ob die Empfehlung zu diesen Aspekten ausbleibt, da keine Evidenz vorliegt oder dies nicht untersucht wurde. Die betrachteten Meta-Analysen und Reviews kommen zu ähnlichen Ergebnissen, wobei sie häufig auf eine unzureichende Datengrundlage verweisen [27, 31, 35, 37]. Dies ist möglicherweise der Grund für das Fehlen von Behandlungsempfehlungen in einigen Leitlinien zur Rezidivprophylaxe und/oder zum Schweregrad der Erkrankung. In jedem Fall ist eine weitere Untersuchung von rezidivprophylaktischen Strategien bei Altersdepressionen angezeigt.

Bei refraktären Depressionen ist die Augmentation mit einem weiteren Wirkstoff eine Behandlungsoption, wobei diese in keiner Leitlinie empfohlen wird und die Evidenzgrundlage sehr schwach ist [18]. Besonders häufig wird eine Augmentation mit Lithium betrachtet, die in den untersuchten Reviews und MetaAnalysen durchaus als wirksam eingestuft wird [37, 40]. Jedoch wird auch dort auf die eingeschränkte Datengrundlage verwiesen. Die in der VA/DoD angedeutete Möglichkeit der Augmentation mit Psychostimulanzien für körperlich schwer erkrankte depressive Ältere könnte auch für die körperlich nicht oder kaum erkrankten depressiven Älteren eine Behandlungsmöglichkeit darstellen [16]. Dies wird neben Lithium- und Neuroleptikaaugmentation auch in der Übersichtsarbeit von Maust, Oslin und Thase untersucht, jedoch ohne eindeutiges Ergebnis [40]. Augmentationsstrategien bei der Behandlung von Altersdepression benötigen weitere spezifische Erforschung, um eine evidenzbasierte Empfehlung ableiten zu können.

Diese Arbeit vergleicht sieben qualitativ hochwertige aktuelle Leitlinien, die aus diversen Versorgungssystemen stammen und unterschiedlich ausführlich auf Altersdepressionen eingehen, und fasst diese zusammen. Dabei fällt die uneinheitliche oder nicht spezifizierte Abgrenzung der Depression im Alter von der Depression im Erwachsenenalter auf. In zukünftigen Studien und Leitlinien wäre eine genauere Differenzierung verschiedener Altersgruppen (z. B. Erwachsene: bis 65; ältere Erwachsene: von 65 bis 80; sehr alte Erwachsene: über 80) sinnvoll. Die mögliche Relevanz einer differenzierteren Betrachtung des höheren Lebensalters zeigt sich in der Meta-Analyse von Tedeschini et al. (2011), in der die Effektivität von diversen Antidepressiva für Patienten zwischen 55 und 65 Jahren nachgewiesen werden konnte, nicht aber für Patienten über 65 Jahre [25].

\section{Take Home Message}

Antidepressive Medikamente sind zur Behandlung von Depressionen im Alter geeignet und werden von der deutschen Leitlinie (DGPPN) und internationalen Leitlinien empfohlen.

- Die Wahl des Antidepressivums sollte durch die bestehende Symptomatik, die körperlichen Erkrankungen, die bereits verschriebenen Medikamente und die Nebenwirkungsprofile der Antidepressiva bestimmt werden.

- Die Behandlung soll mit niedriger Dosis begonnen und die Dosis langsam gesteigert werden.

- Die Behandlung mit Serotoninwiederaufnahmehemmern wird aufgrund des geringeren Nebenwirkungsrisikos unter Beachtung von Hyponatriämie empfohlen.

- Zur Rezidivprophylaxe wird eine zwölfmonatige Stabilisierungsphase bei gleichbleibender Dosierung empfohlen. 


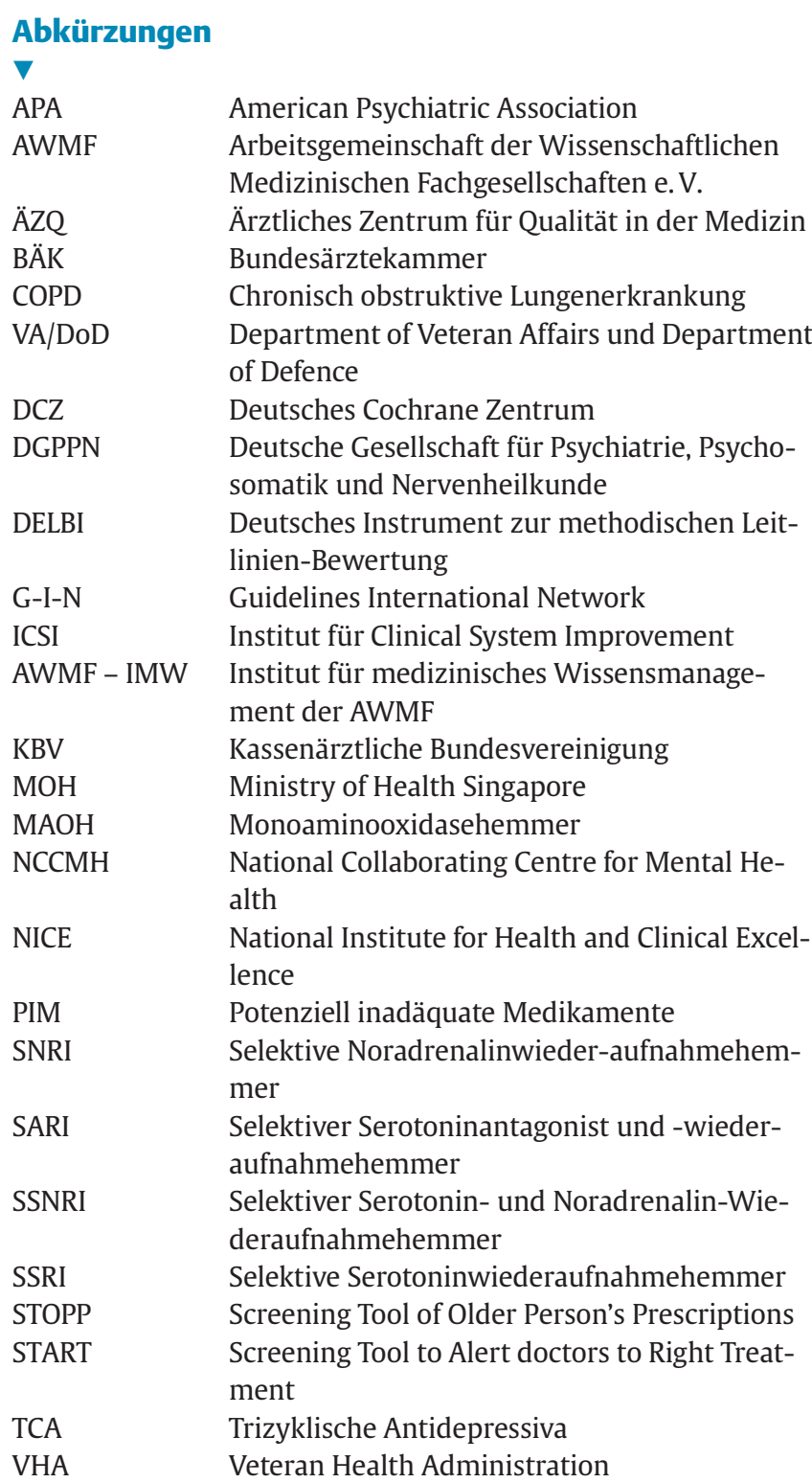

\section{Acknowledgement}

Der Beitrag entstand im Rahmen der Projekte AgeMooDe (BMBFFörderkennzeichen: 1GY1155A) und AgeMooDe+Synergie (BMG/ BVA-Fördernummer: II A 5 - 2513 FSB 014).

Interessenkonflikt: Die Autoren geben an, dass kein Interessenkonflikt besteht.

\section{Literatur}

1 https://www.destatis.de/DE/Publikationen/Thematisch/Bevoelkerung/ VorausberechnungBevoelkerung/BevoelkerungDeutschland2060Presse 5124204099004.pdf?_blob=publication File Zugegriffen am 14.07.2014

2 https://www.destatis.de/GPStatistik/servlets/MCRFileNodeServlet/ DEMonografie_derivate_00000153/Gesundheit_und_Krankheit_im_Alter.pdf Zugegriffen am 14.07.2014

3 Haupt M, Gutzmann H, Wolter D. Psychische Störungen im höheren Lebensalter. In: Möller HJ, Laux G, Kapfhammer HP Hrsg. Psychiatrie, Psychosomatik, Psychotherapie. Band 2: Spezielle Psychiatrie. 4. Aufl. Berlin: Springer; 2011: 1444-1465
4 Luppa M, Sikorski C, Luck T et al. Age- and gender-specific prevalence of depression in latest-life. Systematic review and meta-analysis. J Affect Disord 2012; 136: 212-221

5 Weyerer S, Eifflaender-Gorfer S, Köhler L et al. Prevalence and risk factors for depression in non-demented primary care attenders aged 75 years and older. J Affect Disord 2008; 111: 153-163

6 Luppa M, Heinrich S, Matschinger $H$ et al. Direct costs associated with depression in old age in Germany. Int J Geriatr Psychiatry 2008; 10: $275-282$

7 Baldwin RC, Anderson D, Black S et al. Guideline for the management of late-life depression in primary care. Int J Geratr Psychiatry 2003; 18 : 829-838

8 Diniz BS, Nunes PV, Machado-Vieira $R$ et al. Current pharmacological approaches and perspectives in the treatment of geriatric mood disorders. Curr Opin Psychiatry 2011; 24: 473-477

9 Fiske A, Leobach Weherell J, Gatz M. Depression in Older Adults. Annu Rev Clin Psychol 2009; 5: 363-389

10 Mottram PG, Wilson K, Strobl JJ. Antidepressants for depressed elderly. Cochrane Database of Systematic Reviews 2006; 1: CD003491. DOI: $10.1002 / 14651858 . C D 003491 . p u b 2$

11 Alexopoulos GS. the PROSPECT Group. Interventions for depressed elderly primary care patients. Int J Geriatr Psychiatry 2001; 16: 553 559

12 Rapp MA. Antidepressive Pharmakotherapie im Alter - was ist evidenzbasiert. Pharmakotherapie 2010; 17: 69 - 75

13 Gartlehner MD, Hansen RA, Morgan LC et al. Second-generation antidepressants in the pharmacological treatment of adult depression: An update of the 2007 comparative effectiveness review. Agency for Healthcare Research and Quality (AHRQ) 2011, Verfügbar unter: http:// www.ahrq.gov

14 Beyer JL. Managing depression in geriatric populations. Annals of Clinical Psychiatry 2007; 19: $221-228$

15 http://www.aezq.de/mdb/edocs/pdf/literatur/manual-literaturrecherche.pdf Zugegriffen am 30.03.2014

16 Department of Veterans Affairs/Department of Defense(VA/DoD). Clinical Practice Guideline: Management of Major Depressive Disorder (MDD). Verfügbar unter: http://www.healthquality.va.gov/mdd/mdd_full09_c. pdf 2009

17 National Collaborating Centre for Mental Health (NCCMH), National Institute for Health and Clinical Excellence (NICE). Depression in adults with a chronic physical health problem. Treatment and Management. National Clinical Practice Guideline 91. London: The British Psychological Society and The Royal College of Psychiatrists Leicester 2009

18 National Collaborating Centre for Mental Health (NCCMH), National Institute for Health and Clinical Excellence (NICE). Depression. The NICE Guideline on the Treatment and Management of Depression in Adults (Updated Edition). National Clinical Practice Guideline 90. London: The British Psychological Society and The Royal College of Psychiatrists; 2009

19 American Psychiatric Association (APA). Hrsg Practice Guideline for the Treatment of Patients with Major Depressive Disorder; 2010, Verfügbar unter: http://www.psychiatryonline.org 3. Aufl

20 http://www.moh.gov.sg/cpg Zugegriffen am 29.04.2014

21 Deutsche Gesellschaft für Psychiatrie, Psychotherapie und Nervenheilkunde (DGPPN), Bundesärztekammer (BÄK), Kassenärztliche Bundesvereinigung (KBV), Arbeitsgemeinschaft der Wissenschaftlichen Medizinischen Fachgesellschaften e.V. (AWMF). Hrsg. Nationale Versorgungsleitlinie Unipolare Depression. Berlin: Springer; 2012

22 Mitchell J, Trangle M, Degnan B et al. Adult Depression in Primary Care; 2013, Verfügbar unter: http://www.icsi.org

23 Quaseem A, Forland F, Macbeth $F$ et al. Guidelines International Network: Toward international standards for clinical practice guidelines. Ann Intern Med 2012; 156: 525- 531

24 AWMF - Arbeitsgemeinschaft der Wissenschaftlichen Medizinischen Fachgesellschaften e.V. und ÄZQ - Ärztliches Zentrum für Qualität in der Medizin., Hrsg. Deutsches Instrument zur methodischen Leitlinien-Bewertung (DELBI); 2008, Verfügbar unter: http://www.leitlinien. de/leitlinienmethodik/leitlinienbewertung/delbi/dokumente

25 Tedeschini E, Levkovitz Y, Iovieno $N$ et al. Efficacy of antidepressants for late-life depression: a meta-analysis and meta-regression of placebocontrolled randomized trials. J Clin Psychiatry 2011; 72: 1660-1668

26 Kok RM, Nolen WA, Heeren TJ. Efficacy of treatment in older depressed patients: a systematic review and meta-analysis of double-blind randomized controlled trials with antidepressants. J Affect Disord 2012; 141: $103-115$ 
27 Kok RM, Heeren TJ, Nolen WA. Continuing treatment of depression in the elderly: a systematic review and meta-analysis of double-blinded randomized controlled trials with antidepressants. Am J Geriatr Psychiatry 2011; 19: 249-255

28 Oakes TM, Katona C, Liu P et al. Safety and tolerability of duloxetine in elderly patients with major depressive disorder: a pooled analysis of two placebo-controlled studies. Int Clin Psychopharmacol 2013; 28 : $1-11$

29 Seitz DP, Gill SS, Conn DK. Citalopram versus other antidepressants for late-life depression: a systematic review and meta-analysis. International Journal of Geriatric Psychiatry 2010; 25: 1296-1305

30 Pizzi C, Rutjes AWS, Costa GM et al. Meta-analysis of selective serotonin reuptake inhibitors in patients with depression and coronary heart disease. Am J Cardiol 2011; 107: 972 - 979

31 Wilkinson $P$, Izmeth $Z$. Continuation and maintenance treatments for depression in older people. Cochrane Database Syst Rev 2012; 11: CD006727

32 Del Casale A, Giradi P, Brugnoli R et al. Duloxetine in the treatment of elderly people with major depressive disorder. Riv Psichiatr 2012; 4786: $479-488$

33 Dhillon S. Duloxetine: a review of its use in the management of major depressive disorder in older adults. Drugs Aging 2013; 30: 59-79

34 Fagiolini A, Comandini A, Dell'Osso MC et al. Rediscovering trazodone for the treatment of major depressive disorder. CNS Drugs 2012; 26: $1033-1049$

35 Katona C, Bindman DC, Katona CP. Antidepressants for older people: What can we learn from the current evidence base? Maturitas 2014; 79: $174-178$

36 Mitchell PB, Harvey SB. Depression and the older medical patient when and how to intervene. Maturitas 2014; 79: 153-159
37 Cooper C, Katona C, Lyketsos K et al. A systematic review of treatments for refractory depression in older people. Am J Psychiatry 2011; 168: $681-688$

38 Dolder C, Nelson M, Stump A. Pharmacological and clinical profile of newer antidepressants: Implications for the treatment of elderly patients. Drugs Aging 2010; 27: 625-640

39 Holland J, Bhogle M. Sertraline and mirtazapine as geriatric antidepressants. Psychiatria Danubia 2013, (Suppl 2): 286-290

40 Maust DT, Oslin DW, Thase ME. Going beyond antidepressant monotherapy for incomplete response in non-psychotic late-life depression: A critical review. Am J Geriatr Psychiatry 2013; 21: 973-986

41 Howland $R H$. A benefit-risk assessment of agomelatine in the treatment of major depression. Drug Saftey 2011; 34: 709-731

42 Jaffe RJ, Novakovic V, Peselow ED. Scopolamine as an antidepressant: A systematic review. Neuropharmacology 2013; 36: 24-26

43 Boyce RD, Hanlon JT, Karp JF et al. A review of the effectiveness of antidepressant medications for depressed nursing home residents. J Am Med Dir Assoc 2012; 13: 326-331

44 Holt S, Schmiedl S, Thürmann AP. Potenziell inadäquate Medikation für ältere Menschen: Die PRISCUS-Liste. Deutsches Ärzteblatt 2010; 31 32: $543-551$

45 http://www.americangeriatrics.org/files/documents/beers/2012AGSBeersCriteriaCitations.pdf Zugegriffen am 29.05.2015

46 O'Mahony D, O'Sullivan D, Byrne $S$ et al. STOPP/START criteria for potentially inappropriate prescribing in older people: version 2 . Age and Ageing 2014; 0: $1-6$

47 Gallagher P, Rayn C, Byrne S et al. STOPP (Screening Tool of Older Person's Prescriptions) and START (Screening Tool to Alert doctors to Right Treatment). Consensus validation. Int J Clin Pharmacol Ther 2008; $46: 72-83$ 


\section{Literaturangaben der ausgeschlossenen Leitlinien}

$\nabla$

Erscheinungszeitraum nicht aktuell (5)

- Anderson IM, Ferrier IN, Baldwin RC et al. Evidence-based guidelines for treating depressive disorders with antidepressants: A revision of the 2000 British Association for Psychopharmacology guidelines. J Psychopharmacol 2008; 22: 343 - 396

- Fulcher CD, Badger T, Gunter AK et al. Putting Evidence into Practice ${ }^{\circledR}$ : Interventions for depression. Clin J Oncol Nurs 2008; $12: 131$ - 140

- New Zealand Guidelines Group, Hrsg. Identification of common mental disorders and management of depression in primary care. An evidence-based best practice guideline. Wellington: Ministry of Health; 2008

- Quaseem A, Snow V, Denberg TD et al. Using second-generation antidepressants to treat depressive disorders: A clinical practice guideline from the American college of physicians. Ann Intern Med 2008; 149: 725 - 733

- Working Group on the Management of Major Depression in Adults. Clinical practice guideline on the management of major depression in adults. Madrid: National Plan for the SHN of the MHCA, avalia-t; 2008

\section{Population entspricht nicht der Zielgruppe (2)}

- Michigan Quality Improvement Consortium Guideline. Primary care diagnosis and management of adults with depression. 2014; Verfügbar unter: http://mqic.org/pdf/mqic_primary_ care_diagnosis_and_management_of_adults_with_depression_cpg.pdf

- Austin MP, Highet $\mathrm{N}$ and the Guidelines Expert Advisory Committee. Clinical practice guidelines for depression and related disorders - anxiety, bipolar disorder and puerperal psychosis in the perinatal period. A guideline for primary care health professionals. Melbourne: beyondblue, the national depression initiative; 2011
Intervention entspricht nicht dem Zielkriterium (3)

- Scottish Intercollegiate Guidelines Network. Non-pharmaceutical management of depression in adults. A national clinical guideline. Edinburgh: Scottish Intercollegiate Guidelines Network. 2010; Verfügbar unter: www.sign.ac.uk

- Harvath TA, McKenzie G. Evidence-based geriatric nursing protocols for best practice. In: Boltz M, Capezuti E, Fulmer T, Zwicker D. Depression in older adults. 4. Aufl. New York: Springer Publishing Company; 2012; 135 - 162

- Registered Nurses Association of Ontario. Caregiving strategies for older adults with delirium, dementia and depression. Supplement. Toronto: Registered Nurses' Association of Ontario; 2012; Verfügbar unter: http://rnao.ca/sites/rnao-ca/files/Caregiving_Strategies_for_Older_Adults_with_Delirium_Dementia_and_Depression.pdf

\section{Methodische Schwäche (3)}

- University of Michigan Health System. Depression. Guidelines for Clinical Care. 2005; Verfügbar unter: http://www.med. umich.edu/1info/fhp/practiceguides/depress/depress.pdf

- Fitzsimmons S, Schönfelder DP. Evidence-based practice guideline. Wheelchair biking for the treatment of depression. J Gerontol Nurs 2011; 37: 8 - 15

- American Medical Directors Association. Depression in the long-term care setting. Columbia (MD): American Medical Directors Association (AMDA); 2011 